\title{
Solvothermal Synthesis of Well-Disperse ZnS Nanorods with Efficient Photocatalytic Properties
}

\author{
Yun Chen, ${ }^{1}$ Rong-hui Yin, ${ }^{1}$ and Qing-sheng $\mathrm{Wu}^{1,2}$ \\ ${ }^{1}$ Department of Chemistry, Tongji University, Shanghai 200092, China \\ ${ }^{2}$ Shanghai Key Laboratory of Molecular Catalysis and Innovative Materials, Fudan University, Shanghai 200433, China
}

Correspondence should be addressed to Qing-sheng Wu, qswu@tongji.edu.cn

Received 12 August 2011; Accepted 1 December 2011

Academic Editor: Xuedong Bai

Copyright ( 2012 Yun Chen et al. This is an open access article distributed under the Creative Commons Attribution License, which permits unrestricted use, distribution, and reproduction in any medium, provided the original work is properly cited.

\begin{abstract}
Well-disperse short-range-ordered ZnS nanorods with efficient photocatalytic property for photodegradation of Rhodamin B have been successfully synthesized through a solvothermal method. Solvent used can be recovered and reused, which makes the route environment-friendly. Dodecylamine was found effective in organizing nanorods to ordered monolayer. Characterization showed that these nanorods were uniform with the diameter of about $3 \mathrm{~nm}$ and length of nearly $30 \mathrm{~nm}$. And it is expected that these monodisperse $\mathrm{ZnS}$ nanorods have potential applications in electroluminescence materials.
\end{abstract}

\section{Introduction}

Over the past decades, nanocrystals which are monodisperse in size and shape have received substantial attention due to their unique optical and physical properties compared to bulk materials [1]. However, it is still a great challenge to fabricate monodisperse nanorods. Among various nanomaterials, semiconductor nanomaterials, especially metal chalcogenide nanomaterials, have been studied in depth due to their optical and electronic properties arising from the quantum confinement effect and large surface area [2]. In this paper, a solvothermal method is used to synthesize highquality $\mathrm{ZnS}$ nanorods, an important wide bandgap ( $E_{g}$ of $3.66 \mathrm{eV}$ at room temperature) II-VI group semiconductor, which is highly desirable in potential applications such as electroluminescence devices, photoluminescence devices, and semiconductor quantum well devices, flat-panel display, infrared windows, sensors, and lasers [3-10].

Various approaches have been explored to pursue a simple and shape-controllable synthetic method for semiconductor monodisperse nanocrystals, including irradiation method, solution-phase synthetic route, and solvothermal synthesis [11-14]. Among these methods, it is well established that synthetic routes utilizing organometallic/nonmetallic precursors enable production of high-quality monodisperse semiconductors, metals, and metal oxide nanocrystal dispersions [15]. Since the first synthesis for monodisperse $\mathrm{ZnS}$ nanoparticles by size selective photocorrosion [16], some similar approaches in synthesizing monodisperse nanoparticles have been reported $[17,18]$. However, seldom effort is devoted to synthesizing $\mathrm{ZnS}$ monodisperse nanorods using a simple and nontoxic solution route without tedious size selection processes [19].

In this paper, a long-chain alkylamine is introduced in the traditional solvothermal method, which is conducted simply by the reaction of zinc sulfate and $S$ powder in nontoxic dodecylamine. Due to the special reactivity under high temperature and high pressure, $S$ powder can be directly used as $S$ sources, which avoids usage of its toxic precursors. In addition, the sample shows good photocatalytic effect on the degradation of Rhodamin B.

\section{Experimental}

2.1. Synthesis of $Z n S$ Nanorods. In a typical synthesis, $0.15 \mathrm{~g}$ $\mathrm{ZnSO}_{4} \cdot 7 \mathrm{H}_{2} \mathrm{O}$ was put into a $25 \mathrm{~mL}$ round bottom flask, then $5 \mathrm{~mL}$ dodecylamine was added, and the mixture was stirred for $3 \mathrm{~h}$ at $80^{\circ} \mathrm{C} .0 .032 \mathrm{~g}$ sulfur powder was put into the mixture quickly and stirred heavily for $10 \mathrm{~min}$. Consequently, the mixture was transferred into a Teflon-lined autoclave of $10 \mathrm{~mL}$ capacity. Then the autoclave was sealed and heated at $200^{\circ} \mathrm{C}$ for $1.5 \mathrm{~h}$ and was left to cool to room temperature 
naturally. The products were collected by filtration, washed with deionized water and absolute ethanol. A white powder was obtained after $3 \mathrm{~h}$ dry at $70^{\circ} \mathrm{C}$ in a vacuum drying oven and was preserved in absolute ethanol for further characterization.

2.2. Synthesis of $\mathrm{ZnS}$ Microspheres. $0.15 \mathrm{~g} \mathrm{ZnSO} \cdot \cdot 7 \mathrm{H}_{2} \mathrm{O}$ and $0.032 \mathrm{~g}$ sulfur powder were mixed in water, and then the mixture was stirred and transferred into a Teflon-lined autoclave of $10 \mathrm{~mL}$ capacity. The autoclave was sealed and heated at $200^{\circ} \mathrm{C}$ for $1.5 \mathrm{~h}$ and was left to cool to room temperature naturally. The products were collected by filtration, washed with deionized water and absolute ethanol. A white powder was obtained after $3 \mathrm{~h}$ dry at $70^{\circ} \mathrm{C}$ in a vacuum drying oven and was preserved in absolute ethanol for further characterization.

2.3. Characterization. The phase purities of the obtained samples were measured on a Bruker D8-advance X-ray powder diffractometer with $\mathrm{Cu} \mathrm{K}_{\alpha}$ radiation $(\lambda=1.5418 \AA)$. A small amount of products was dispersed in ethanol by ultrasonic treatment for about $5 \mathrm{~min}$, and then one drop of the resulting solution was placed onto a carbon-coated copper grid and dried at room temperature for TEM visualization. Morphology analysis of the samples was conducted with JEOL JEM-1230 transmission electron microscopy (TEM) operated at $80 \mathrm{kV}$. Room temperature photoluminescence (PL) of as-prepared sample was observed under excitation by UV light at $365 \mathrm{~nm}$ with a Perkin-Elmer LS-55 fluorescence spectrophotometer. The UV-Vis absorption spectrum was recorded on an Agilent 8453 UV-visible spectrophotometer.

2.4. Photocatalytic Activity Measurement. Seven cylindrical Pyrex flasks (capacity ca. $10 \mathrm{~mL}$ ) were used as the photoreactor vessels. The reaction system containing Rhodamine $\mathrm{B}$ $\left(\mathrm{C}_{28} \mathrm{H}_{31} \mathrm{~N}_{2} \mathrm{O}_{3} \mathrm{Cl}\right.$ ) (Sigma-Aldrich Chemical Co.; $1 \times 10^{-4} \mathrm{M}$, $10 \mathrm{~mL})$ and $\mathrm{ZnS}$ nanorods as catalyst $(10 \mathrm{mg})$ was magnetically stirred in the dark for $12 \mathrm{~h}$ to reach the adsorption equilibrium of Rhodamine $\mathrm{B}$ with the catalyst and then exposed to light from a Xujiang middle-pressure Hg lamp $(300 \mathrm{~W})$, and the maximum intensity of the light irradiated on the solution is $365 \mathrm{~nm}$. UV-Vis absorption spectra were recorded at different intervals to monitor the reaction.

\section{Results and Discussion}

The crystalline structure and purity were examined by X-ray diffraction analysis (XRD). As shown in Figure 1, all diffraction peaks can be indexed to face-centered cubic Sphalerite ZnS phase (JCPDS 05-0566), with lattice constants of $a=5.406 \mathrm{~nm}$. An obvious broadening can be observed from the XRD curve which may be caused by the small grain size of the sample [20]. No characteristic peaks ascribing to other phases were observed, which indicates high purity of the prepared $\mathrm{ZnS}$ nanorods.

The size and shape of the products were examined by TEM imaging. Figure 2 shows typical images of $\mathrm{ZnS}$ nanorods, which have very narrow size distributions. These

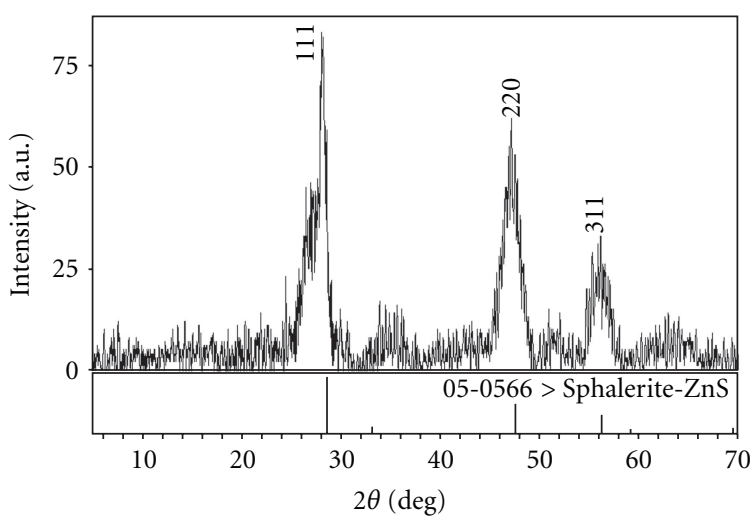

FIGURE 1: XRD curve of $\mathrm{ZnS}$ nanorods.

nanorods have diameters of about $3 \mathrm{~nm}$ and length of nearly $30 \mathrm{~nm}$. The shape and size are uniform and it is observed that all the nanorods are well dispersed. These nanorods can be used as conducting wires in microelectronic devices. Keeping other reaction conditions the same, the morphology of the sample is sphere when dodecylamine is not added (shown in Figure 2(c)). The diameter of these spheres is nearly $2 \mu \mathrm{m}$. It was found that dodecylamine played important role in the formation and dispersion of $1 \mathrm{D} \mathrm{ZnS}$ nanorods. Dodecylamine is long-chain molecule with $-\mathrm{NH}_{2}$. The $\mathrm{N}$ atom has strong coordination ability, which could coordinate with $\mathrm{Zn}^{2+}$, which will control the nucleation velocity of $\mathrm{ZnS}$. Consequently, it limits the crystal growth at high temperature and direct forming well-disperse $\mathrm{ZnS}$ nanorods.

In order to investigate the optical properties of the products, UV-Vis absorption spectrum was also studied as shown in Figure 3. The as-obtained nanorods have the maximum absorption at $269 \mathrm{~nm}$. There is about a $70 \mathrm{~nm}$ blue shift from the bulk counterparts $\left(E_{g}\right.$ of $\left.3.66 \mathrm{eV}\right)$. That may contribute to the quantum size effect of nanomaterials caused by the photogenerated electron-hole pairs [21].

As we all know, the study of luminescence properties can shed some light on defects in the $\mathrm{ZnS}$ crystals and their potential as photonic materials. Figure 4 shows the room-temperature photoluminescence spectrum of the sample excited with UV-light at $365 \mathrm{~nm}$. The slight difference was also observed in the PL spectrum. The band position centered at about $410 \mathrm{~nm}$ is the stable and strong purple emission. This broad emission peak may be due to the band to band transition of $\mathrm{ZnS}$.

It is well known that $\mathrm{ZnS}$ has been used as a semiconductor-type photocatalyst for the photoreductive dehalogenation of halogenated benzene derivatives, photocatalytic degradation of water pollutants, and photocatalytic reduction of toxic metal ions $[22,23]$. To demonstrate the potential applicability of as-synthesized $\mathrm{ZnS}$ nanorods in these applications, their photocatalytic activity by choosing photodegradation of Rhodamin B at room temperature is investigated. The characteristic absorption of Rhodamin B at about $553 \mathrm{~nm}$ is chosen as the monitored parameter for the photo-degradation process. 


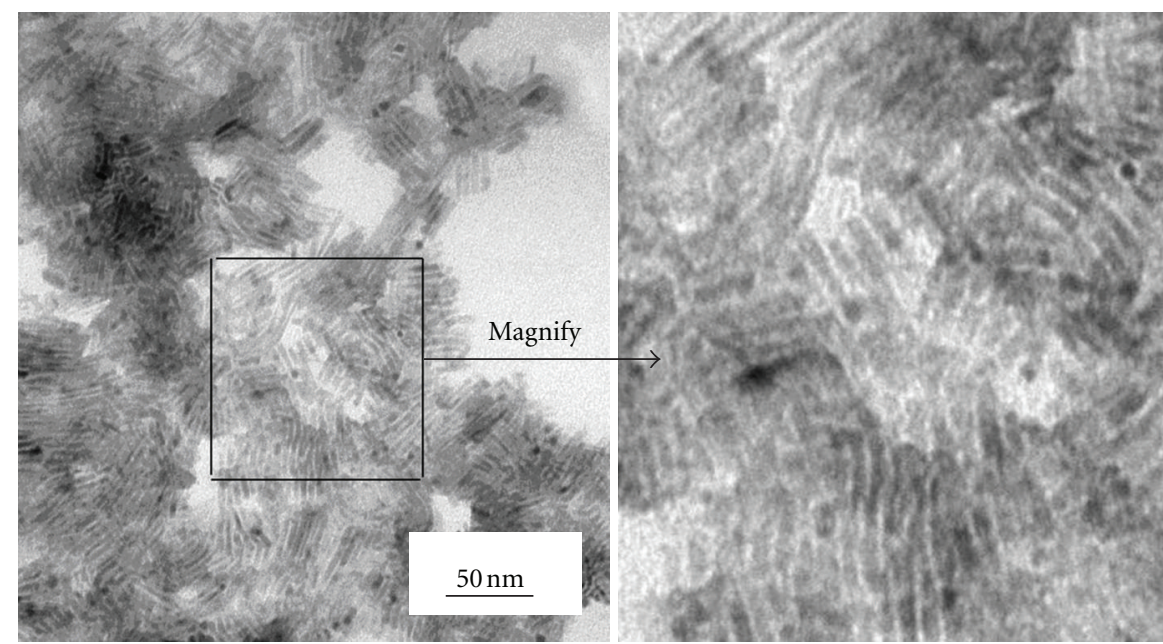

(a)

(b)

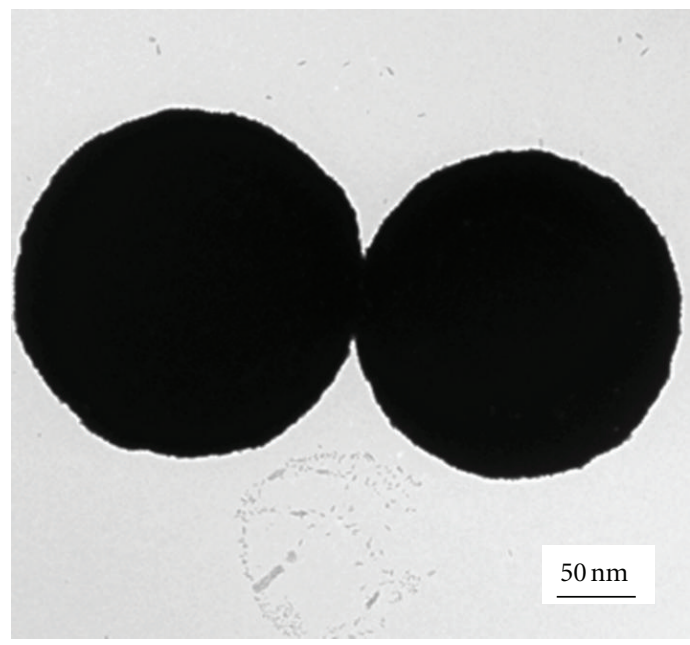

(c)

Figure 2: (a), (b) Typical TEM image of well-disperse ZnS nanorods synthesized with dodecylamine as template. (c) TEM image of ZnS spheres obtained without dodecylamine.

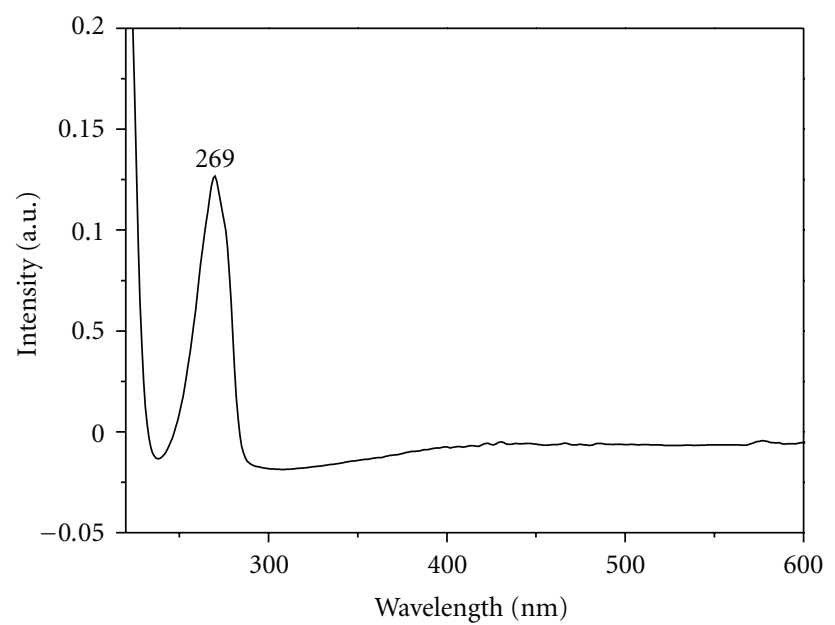

FIGURE 3: UV-Vis absorption spectrum of as-prepared $\mathrm{ZnS}$ nanorods. 


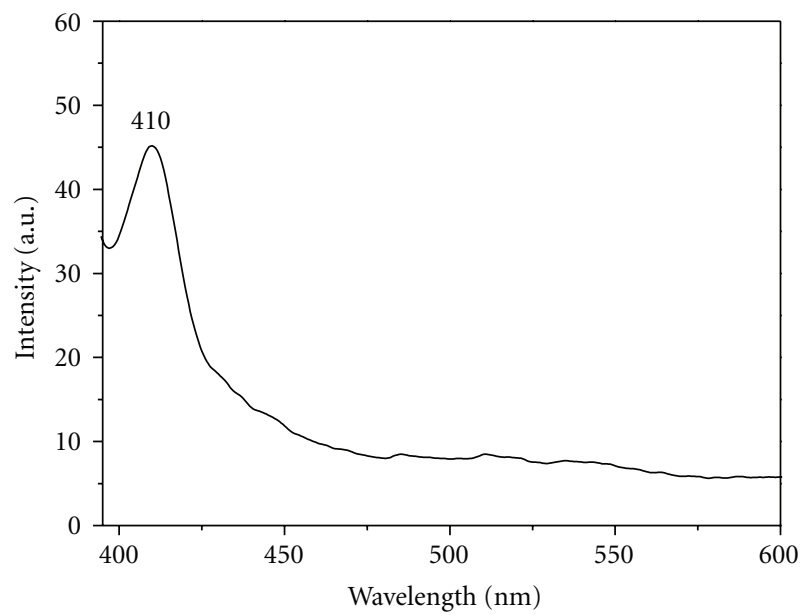

FIGURE 4: PL emission spectrum of as-prepared ZnS nanorods.

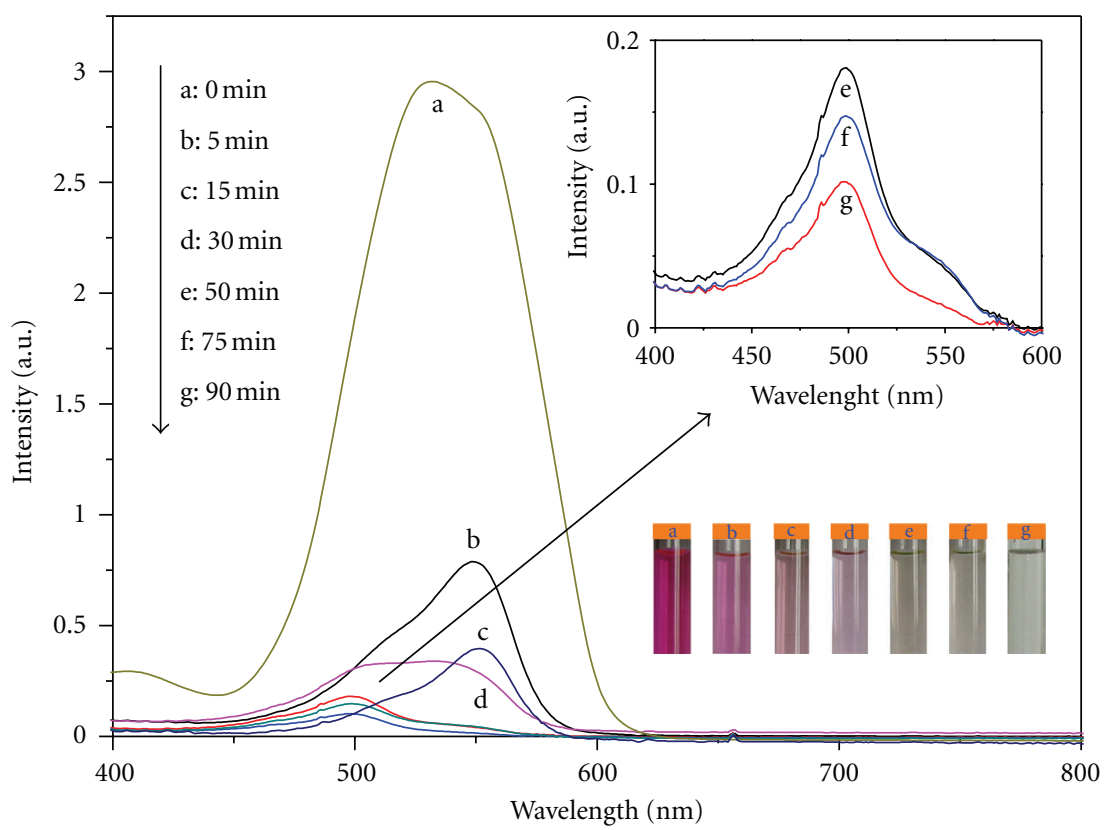

Figure 5: Time-dependent absorption spectra of a solution of Rhodamin B $\left(1 \times 10^{-4} \mathrm{~mol} / \mathrm{L}, 10 \mathrm{~mL}\right)$ in the presence of monodisperse ZnS nanorods after its exposure to UV-light for (a) 0, (b) 5, (c) 15, (d) 30, (e) 50, (f) 75, and (g) 90 min. The inset (upper part) shows the enlarged temporal evolution of absorption $(\mathrm{e}-\mathrm{g})$.

Figure 5 shows the absorption spectra of an aqueous solution of Rhodamin B (initial concentration: $1.0 \times$ $10^{-4} \mathrm{~mol} / \mathrm{L}, 10 \mathrm{~mL}$ ) in the presence of $10 \mathrm{mg} \mathrm{ZnS}$ nanorods under exposure to UV-light for different time. The absorption peaks almost diminish gradually as the exposure time increases and completely disappear after about $50 \mathrm{~min}$. The results suggest that well-disperse $\mathrm{ZnS}$ nanorods can adsorb the dye molecules efficiently. A series of color changes (insert down part in Figure 5) corresponding to the sequential changes are detected by UV-Vis absorption measurements.

In order to exclude the contribution of the decomposition of Rhodamin B itself, a test of the decomposition of $\mathrm{RhB}$ itself under light irradiation without photocatalyst was performed. The result was shown in Figure 6. It indicated that
RhB itself was not decomposed in 180 min under the same light irradiation.

As illustrated in Figure 7, we plot the degradation rate under different exposure time. Curve a shows degradation rate of Rhodamin B by as-synthesized nanorods. It is obvious that degradation rate is high at the first $5 \mathrm{~min}$, indicating high catalytic activity. After exposed about $15 \mathrm{~min}$, the conversion reaches nearly $90 \%$, which is better than that of $\mathrm{TiO}_{2}$ as reported by Shao et al. [24]. With the time extending to $90 \mathrm{~min}$, the conversion is $98 \%$, indicating the nearly complete degradation. However, in the latter $75 \mathrm{~min}$, the conversion increases only about $8 \%$.

In comparison with the effect of spheres (curve b in Figure 7), the performance of nanorods is much better. 


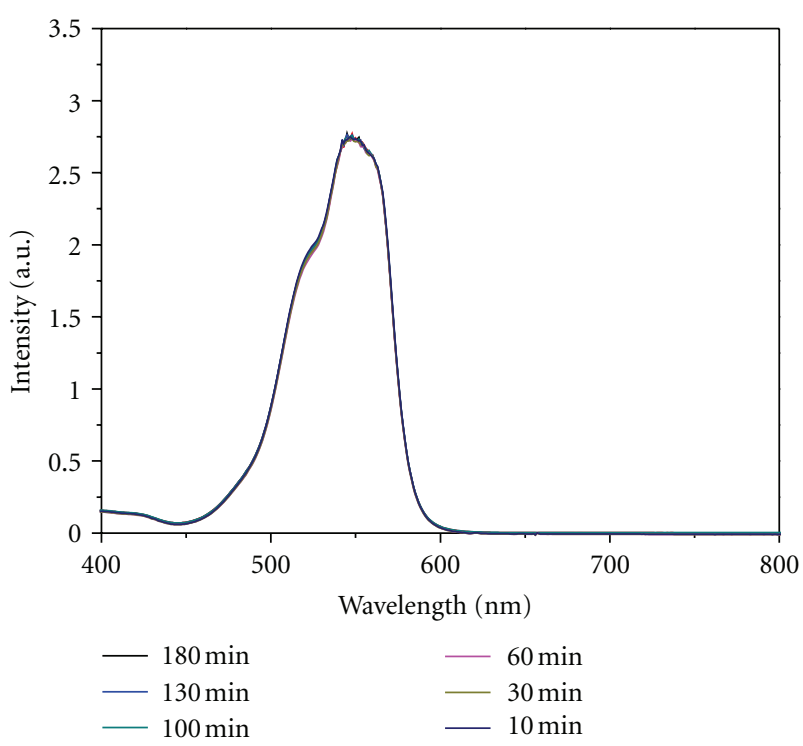

Figure 6: Time-dependent absorption spectra of a solution of Rhodamin B $\left(1 \times 10^{-4} \mathrm{~mol} / \mathrm{L}, 10 \mathrm{~mL}\right)$ without $\mathrm{ZnS}$ catalyst after its exposure to UV-light for $180 \mathrm{~min}$.

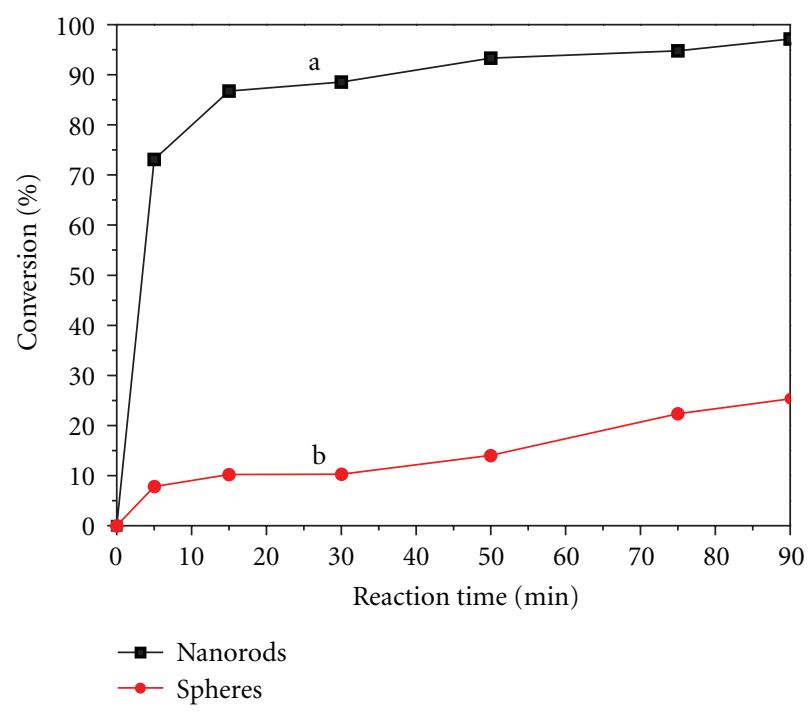

Figure 7: Degradation rate of Rhodamin B.

Dodecylamine used in the reaction is an effective stabilizer which prevents small nanorods from aggregation. In the degradation of $\mathrm{RhB}$, the reaction was performed in the surface of photocatalyst and the reaction temperature is higher than room temperature. So the degradation rate can be reduced if the aggregation happens. It is believed that onedimensional nanostructure can avoid aggregation at higher temperature; therefore well-disperse nanorods could provide much more activation site which can accelerate the degradation efficiently.

\section{Conclusion}

In conclusion, monodisperse $\mathrm{ZnS}$ nanorods have been synthesized with a solvothermal method. Instead of using expensive and toxic organometallic/nonmetallic precursors, the simple reaction between zinc sulfate and sulfur powders in nontoxic solvent suggests a safe and cheap route for monodisperse nanorods. These well-dispersed nanorods exhibit high photocatalytic activity, which makes it useful in the treatment of polluted water.

\section{Acknowledgments}

The authors are grateful to the financial support of the National Natural Science Foundation (nos. 21101118, 51072134, 91122025) of China, the State Major Research Plan (973) of China (no. 2011CB932404), the Fundamental Research Funds for the Central Universities, the Program for Young Excellent Talents in Tongji University (no. 2008KJ036), and the Shanghai Key Laboratory of Molecular Catalysis and Innovative Materials (no. 2012MCIMKF03).

\section{References}

[1] J. Xu, J. P. Ge, and Y. D. Li, "Solvothermal synthesis of monodisperse PbSe nanocrystals," Journal of Physical Chemistry B, vol. 110, no. 6, pp. 2497-2501, 2006.

[2] W. Wang, L. Chen, S. Wang et al., "Low-temperature synthesis of nearly monodisperse ZnS nanospheres using a facile solution-phase approach," Australian Journal of Chemistry, vol. 59, no. 11, pp. 791-795, 2006.

[3] H. Yang, P. H. Holloway, and B. B. Ratna, "Photoluminescent and electroluminescent properties of $\mathrm{Mn}$-doped $\mathrm{ZnS}$ nanocrystals," Journal of Applied Physics, vol. 93, no. 1, pp. 586-592, 2003.

[4] Y. R. Do, Y. C. Kim, S. H. Cho, J. H. Ahn, and J. G. Lee, "Improved output coupling efficiency of a $\mathrm{ZnS}: \mathrm{Mn}$ thin-film electroluminescent device with addition of a two-dimensional $\mathrm{SiO}_{2}$ corrugated substrate," Applied Physics Letters, vol. 82, no. 23, pp. 4172-4174, 2003.

[5] C. N. Xu, T. Watanabe, M. Akiyama, and X. G. Zheng, "Preparation and characteristics of highly triboluminescent $\mathrm{ZnS}$ film," Materials Research Bulletin, vol. 34, no. 10-11, pp. 14911500, 1999.

[6] W. Chen, Z. Wang, Z. Lin, and L. Lin, "Thermoluminescence of ZnS nanoparticles," Applied Physics Letters, vol. 70, no. 11, pp. 1465-1467, 1997.

[7] J. Liu, Z. Guo, W. Wang, Q. Huang, K. Zhu, and X. Chen, "Heterogeneous ZnS hollow urchin-like hierarchical nanostructures and their structure-enhanced photocatalytic properties," Nanoscale, vol. 3, no. 4, pp. 1470-1473, 2011.

[8] C. Bi, L. Pan, Z. Guo et al., "Facile fabrication of wurtzite ZnS hollow nanospheres using polystyrene spheres as templates," Materials Letters, vol. 64, no. 15, Article ID 11232, pp. 16831681, 2010.

[9] T. V. Prevenslik, "Acoustoluminescence and sonoluminescence," Journal of Luminescence, vol. 87, pp. 1210-1212, 2000.

[10] S. Padalkar, J. Hulleman, S. M. Kim et al., "Fabrication of ZnS nanoparticle chains on a protein template," Journal of Nanoparticle Research, vol. 11, no. 8, pp. 2031-2041, 2009.

[11] X. Xue, J. Chen, and Y. Hu, "Preparation of well uniform-sized and monodisperse ZnS nanoballs by $\gamma$-irradiation method," Materials Letters, vol. 61, no. 1, pp. 115-118, 2007.

[12] C. B. Murray, D. J. Norris, and M. G. Bawendi, "Synthesis and characterization of nearly monodisperse $\mathrm{CdE}(\mathrm{E}=\mathrm{S}, \mathrm{Se}, \mathrm{Te})$ 
semiconductor nanocrystallites," Journal of the American Chemical Society, vol. 115, no. 19, pp. 8706-8715, 1993.

[13] A. M. Morales and C. M. Lieber, "A laser ablation method for the synthesis of crystalline semiconductor nanowires," Science, vol. 279, no. 5348, pp. 208-211, 1998.

[14] X. Peng, L. Manna, W. Yang et al., "Shape control of CdSe nanocrystals," Nature, vol. 404, no. 6773, pp. 59-61, 2000.

[15] X. Wang, J. Zhuang, Q. Peng, and Y. Li, "A general strategy for nanocrystal synthesis," Nature, vol. 437, no. 7055, pp. 121-124, 2005.

[16] Y. Ohko, M. Setani, T. Sakata, H. Mori, and H. Yoneyama, "Preparation of monodisperse $\mathrm{ZnS}$ nanoparticles by size selective potocorrosion," Chemistry Letters, no. 7, pp. 663-664, 1999.

[17] Y. Zhang and Y. Li, "Synthesis and characterization of monodisperse doped ZnS nanospheres with enhanced thermal stability," Journal of Physical Chemistry B, vol. 108, no. 46, pp. 17805-17811, 2004.

[18] Y. Zhao, W. Shi, M. Gong, F. Yu, and T. Chen, "A $\gamma$-ray irradiation route to fabricate monodisperse zinc sulfide hollow spheres using silica as templates," Materials Chemistry and Physics, vol. 94, no. 2-3, pp. 292-297, 2005.

[19] H. Y. Jung, J. Jin, M. P. Hyun et al., "Synthesis of quantumsized cubic $\mathrm{ZnS}$ nanorods by the oriented attachment mechanism," Journal of the American Chemical Society, vol. 127, no. 15, pp. 5662-5670, 2005.

[20] K. Zhang, I. V. Alexandrov, R. Z. Valiev, and K. Lu, "Structural characterization of nanocrystalline copper by means of x-ray diffraction," Journal of Applied Physics, vol. 80, no. 10, pp. 5617-5624, 1996.

[21] P. Calandra, M. Goffredi, and V. T. Liveri, "Study of the growth of $\mathrm{ZnS}$ nanoparticles in water/AOT/n-heptane microemulsions by UV-absorption spectroscopy," Colloids and Surfaces A, vol. 160, no. 1, pp. 9-13, 1999.

[22] I. Tsuji, H. Kato, and A. Kudo, "Visible-light-induced $\mathrm{H}_{2}$ evolution from an aqueous solution containing sulfide and sulfite over a $\mathrm{ZnS}-\mathrm{CuInS}_{2}-\mathrm{AgIn}_{2}$ solid-solution photocatalyst," Angewandte Chemie-International Edition, vol. 44, no. 23, pp. 3565-3568, 2005.

[23] D. Wu, Y. Jiang, Y. Yuan, J. Wu, and K. Jiang, "ZnO-ZnS heterostructures with enhanced optical and photocatalytic properties," Journal of Nanoparticle Research, vol. 13, no. 7, pp. 2875-2886, 2010.

[24] C. Shao, T. Cao, Y. Li, C. Wang, and Y. Liu, "One-step nonaqueous synthesis of pure phase $\mathrm{TiO}_{2}$ nanocrystals from $\mathrm{TiCl}_{4}$ in butanol and their photocatalytic properties," Journal of Nanomaterials, vol. 2011, Article ID 267415, 6 pages, 2011. 

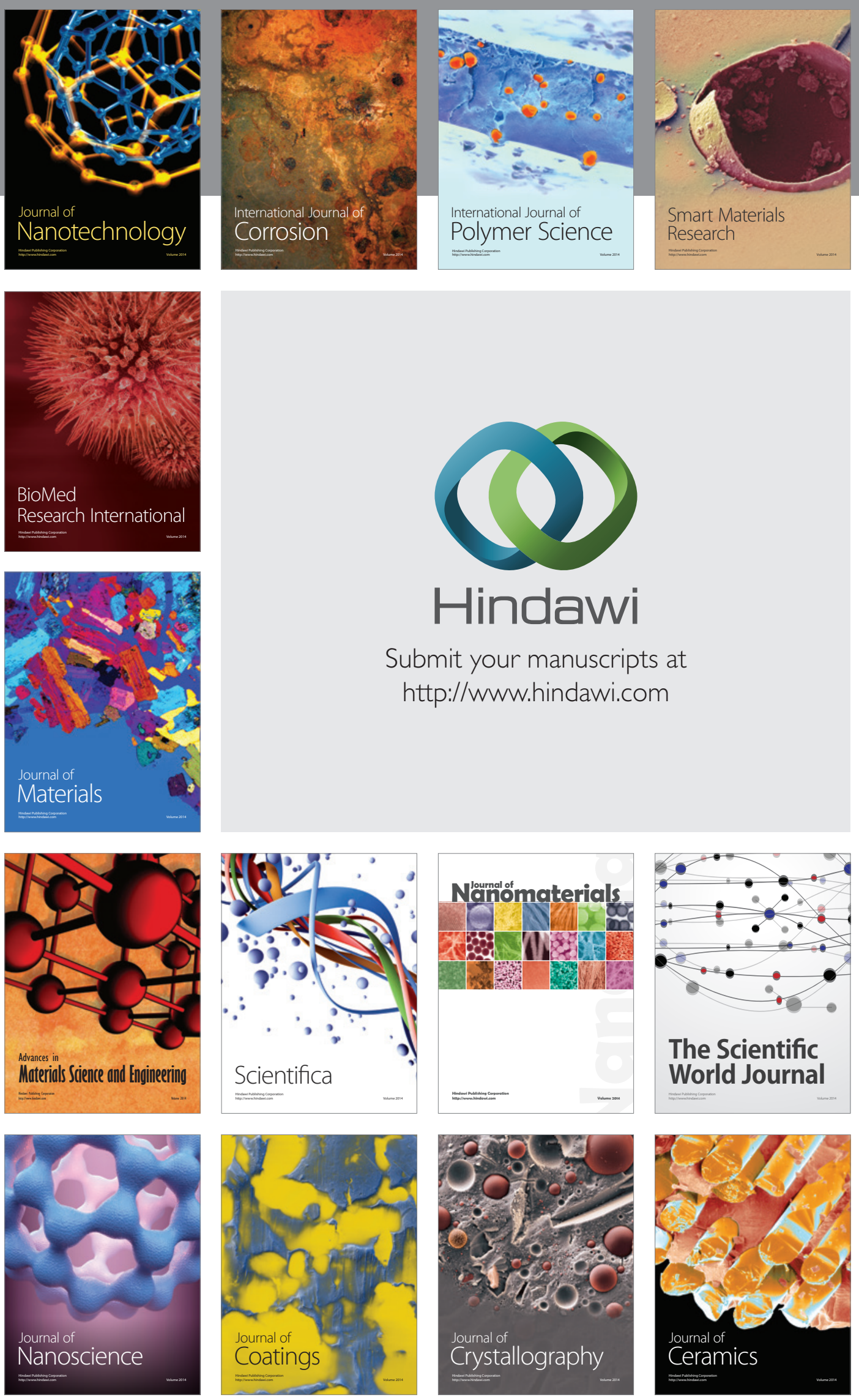

The Scientific World Journal

Submit your manuscripts at

http://www.hindawi.com

\section{World Journal}

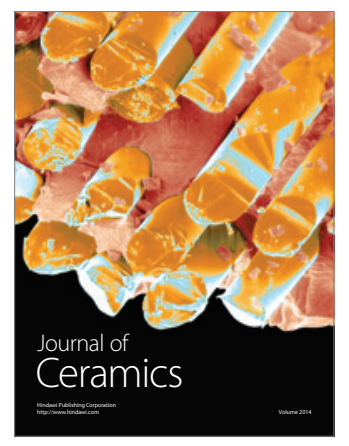

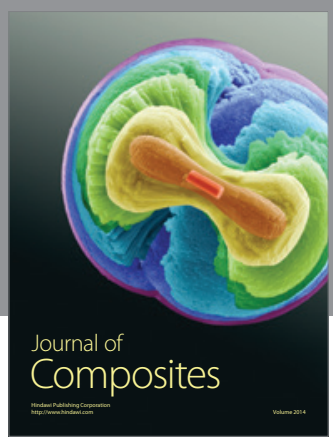
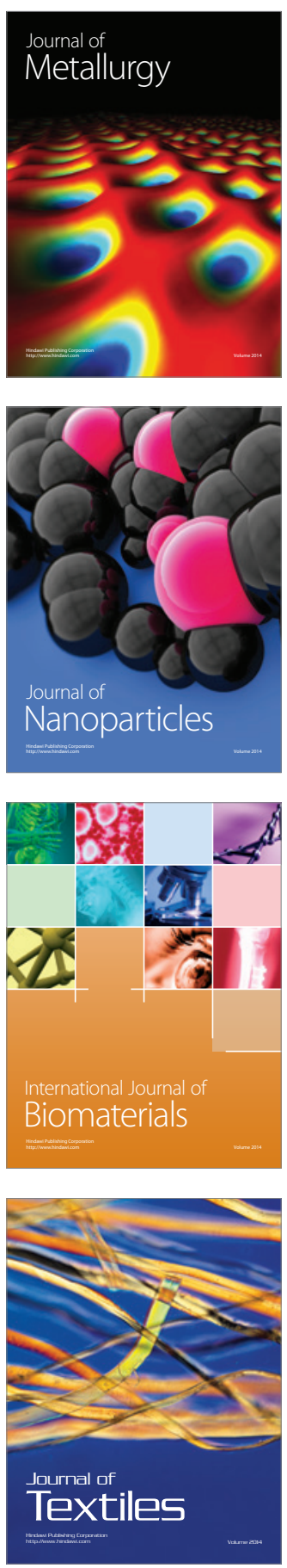